\title{
Lip Changes following Anterior Teeth Retraction in Orthodontically Treated Patients
}

\author{
Karpaga Preethitha .S
}

\begin{abstract}
Aim: To estimate the changes in the lip with the retraction of anterior teeth in orthodontically treated patients. Objective: To study the amount the amount of changes seen in the upper lip with the various degrees of retraction of the upper anterior teeth in patients under orthodontic correction. Background: In orthodontic treatment the correction of anterior teeth proclination is carried out by creating space with extractions. With the retraction of the proclined anterior teeth, the lip which is in close contact with it also undergoes changes based on the degree of retraction. This study aims to analyse the various changes of the lip with the changes in anterior teeth position. Materials and method: Pre- and post-treatment cephalogramsof 41 patients who have undergone correction of class I or class II division I malocclusion is collected and traced manually and analysed cephalometricallyfor this study. Result: Positive correlation between the retractions of the upper lip following the upper teeth retraction is seen in an average of 0.51 cases. Negative correlation i.e. increase in the lip thickness with the retraction of upper anterior teeth is seen in an average of 0.29 cases and no changes are seen after the anterior teeth retraction in average of 0.19 cases.
\end{abstract}

Keywords: Retraction, Premolar Extraction, Chephalogram

\section{Introduction}

It is important to evaluate the social aspects of orthodontic treatment outcomes as well as to establish a functional occlusion (1). Profile improvement via orthodontic treatment is important in evaluating the treatment results.Indeed, orthodontists are frequently questioned about possible facial profile changes created by a particular treatment plan. Since the perioral profile of the lip form is a critical factor in achieving facial aesthetics, it is necessary to evaluate and predict the post-treatment lip position before determining a treatment plan.(2)

In recent years there is a paradigm shift in orthodontic treatment where the importance of treatment has been shifted from hard tissue to soft tissue. During the 70s and 80s concentration of orthodontic treatment was in alining the teeth, decrowding them and retracting them with hardly any importance being given to soft tissues. But at present the importance to soft tissue has taken a front step and is given more preference in treatment than the hard tissues. At the end of treatment almost all the time it is the lips which are more visible and defines the profile and appearance of patient than the teeth. How much changes are brought about with the extraction and retraction of teeth or by moving the teeth inside is a matter of contradiction with various authors giving various values regarding this. So this was a study that was conducted to find out if there is relevant change with the upper lip falling back when compared to after retraction of upper incisors.

\section{Materials and Method}

Pre-operative and post-operative cephalograms of 41 patients of age group ranging from 12-30 years of age were collected. The cases includes both male and female patients with class I and class II division I malocclusions.

The criterion for selection includes:

1) Proclination of upper anterior teeth

2) Protrusion of lip

3) Patients with skeletal class I or class II growth pattern.
4) Patients treated with extraction of premolars for space gaining.

5) Patients treated without use of extra oral anchorage.

6) Cephalograms of patients taken with relaxed lip and teeth in centric occlusion.

The cephalograms are traced manually and are analysed cephalometrically.

The distance between the most prominent point on the upper lip and a reference line drawn at 80-90 degree from the SN plane is measured. Similarly the distance between the tip of the upper incisor and the same reference line is calculated in the pre-operative and post-operative cephalograms. These values are recorded and analysed.

The difference in the distance between the upper lip and the reference line in the preoperative and postoperative cephalograms gives the amount of changes seen in the upper lip position. Similarly the difference in the distance between the tip of the central incisor and the reference line in the preoperative and postoperative cephalograms gives the amount of upper anterior teeth retraction done at the end of the orthodontic treatment.

\section{Statistical procedure}

For each variable the mean, standard deviation and standard mean error is calculated. The differences between the means are calculated using ANNOVA test.

\section{Result}

$\begin{array}{cccccc}\begin{array}{c}\text { Number } \\ \text { of }\end{array} & \text { Standard } & \begin{array}{c}\text { Standard } \\ \text { Error of }\end{array} & & & \\ \text { Group } & \text { Points } & \text { Mean } & \text { Deviation } & \text { Mean } & \text { P value } \\ \text { pre lip } & 41 & 17.415 & 3.301 & 0.5156 & * \mathrm{P}>0.05 \\ \text { post-lip } & 41 & 16.854 & 3.539 & 0.5528 & * * * \mathrm{P}<0.001 \\ \text { teethretr } & 40 & 4.750 & 2.362 & 0.3734 & * * * \mathrm{P}<0.001 \\ \text { lip retrac } & 41 & 0.6098 & 2.854 & 0.4457 & * * * \mathrm{P}<0.001\end{array}$




\section{International Journal of Science and Research (IJSR) \\ ISSN (Online): 2319-7064}

Index Copernicus Value (2015): 78.96 | Impact Factor (2015): 6.391

\begin{tabular}{lcll}
\multicolumn{1}{c}{ Table 2 } & & \\
Comparison & mean & \multicolumn{1}{c}{$q$} & $P$ value \\
\hline pre lip vs post-lip & 0.5610 & 1.177 & $* \mathrm{P}>0.05$ \\
pre lip vs teeth ret & 12.665 & 26.412 & $* * * \mathrm{P}<0.001$ \\
pre lip vs lip retrac & 16.805 & 35.265 & $* * * \mathrm{P}<0.001$ \\
post-lip vs teeth retr & 12.104 & 25.242 & $* * * \mathrm{P}<0.001$ \\
post-lip vs lip retrac & 16.244 & 34.088 & $* * * \mathrm{P}<0.001$ \\
teeth retr vs lip retrac & 4.140 & 8.635 & $* * * \mathrm{P}<0.001$
\end{tabular}

The table 2 shows that there is a significant difference between the preoperative and postoperative position of the lip. Highly significant difference is seen between the preoperative lip position \& teeth retraction and preoperative lip position \& lip retraction.

Postoperative lip position \& teeth retraction and postoperative lip position \&lip retractions also shows highly significant difference.

The table also shows that there is a highly significant difference between the teeth retraction and the lip retraction. This data proves that the upper lip shows highly significant change following the retraction of the upper anterior teeth at the end of the orthodontic treatment.

\section{Discussion}

The determination of outcome of an orthodontic treatment is mainly based on the visible changes in appearance and satisfaction of the patients. This change in the lip position is highly valuable for patients with incompetent lips preoperatively. According to Ramos et al. (2005) upper lip retraction is significantly correlated with maxillary incisor retraction, measured at the cervical point(3). The incisal edge or the most anterior point of the incisor has been used as the most common landmark. However, previous studies revealed that the correlation coefficients using this point have a weaker contribution ratio for the upper lip(2).The study ofTalass et al. (1987) attributed upper lip change at labralesuperious to lip thickness and dental variables $(\mathrm{R}=$ 0.69)(4). Moreover,according toStalpers et al. (2007) found that overjet reduction and initial upper lip thickness could explain 15 per cent of the variation in upper lip position. (5).Therefore, the contribution ratios of the parameters predicting upper lip position in previous investigations were relatively low.A previous study by Yasutomi et al., 2006investigated bimaxillarydentoalveolar protrusion subjects. The ratio of maxillary incisor retraction to upper lip retraction was $1.85: 1(\mathrm{R} 2=0.31)$, while retraction in Class II division 1 cases was 2.22:1 (6).

However according to this study there is no significant relation between the preoperative and postoperative lip positions. This study shows that there is a high significant change in the lip position with the retraction of the teeth which is in accordance with the previous studies. The change in the upper lip can be a change in its position or it can be a change in the thickness of the lip following the space created with the anterior teeth being moved backwards.

\section{Conclusion}

Based on the observations we come to the following conclusions

1) The amount of change in the upper lip following upper teeth retraction varies with each patient.

2) The change in the lip can be exhibited in the form of change in the position of the lip or it can be a change in the thickness of the lip.

The studies on various changes in the soft tissue profile are important to predict the outcome of the orthodontic treatment. This helps to provide a more aesthetic and satisfactory treatment to the patients.

\section{References}

[1] Graber TM, Vanarsdall RL Jr.Sarver DM, Proffit WR, Ackerman JL. Diagnosis and treatment planning in orthodontics. In: Graber TM, Vanarsdall RL Jr., editors. Orthodontics. Current principles and techniques. 3rd edn. St Louis: Mosby Inc; 2000. p. 3-115

[2] Effects of retraction of anterior teeth and initial soft tissue variables on lip changes in Japanese adults HirokoHayashida, Hideki Ioi , ShunsukeNakata , Ichiro Takahashi , Amy L. Counts DOI: http://dx.doi.org/10.1093/ejo/cjq095 419-426 First published online: 21 October 2010

[3] Ramos AL, Sakima MT, dos Pinto AS, Bowman SJ. Upper lip changes correlated to maxillary incisor retraction - a metallic implant study. Angle Orthodontist 2005;75:499-505.

[4] Talass MF, Talass L, Baker RC. Soft-tissue profile changes resulting from retraction of maxillary incisors. American Journal of Orthodontics and DentofacialOrthopedics 1987;91:385-394.

[5] Stalpers MJP, Booij JW, Bronkhorst EM, KuijpersJagtman AM, Katsaros C. Extraction of maxillary first permanent molars in patients with Class II division 1 malocclusion. American Journal of Orthodontics and DentofacialOrthopedics 2007;132:316-323.

[6] Yasutomi H, Ioi H, Nakata S, Nakasima A. Effects of retraction of anterior teeth on horizontal and vertical lip positions in Japanese adults with the bimaxillarydentoalveolar protrusion. Orthodontic Waves 2006;65:141-147. 